\title{
Nutrition and the elderly surgical patients
}

\begin{abstract}
Malnutrition is common among the elderly surgical patients. The surgical injuries to tissues cause a cascade of endocrine and metabolic changes which can impede full recovery after surgery. Active screening for malnutrition, proactive preoperative intervention and nutritional support postoperatively have all been shown to reduce breakdown of muscle mass, reduce catabolism and insulin resistance due to surgical injuries and reduce postoperative complications.
\end{abstract}

Keywords: elderly, malnutrition, insulin resistance, perioperative catabolism, perioperative nutritional support
Volume 4 Issue 5 - 2017

\author{
Si Ching Lim, I Adrian Jit Hin Koh \\ 'Senior Consultant, Changi General Hospital, Department of \\ Geriatric Medicine, Singapore \\ 2Director Head and Neck Surgery, Changi General Hospital, \\ Singapore
}

Correspondence: Si Ching Lim, MB ChB, MRCP (UK), Senior Consultant, Department of Geriatric Medicine, Changi General Hospital, Singapore, Email si_ching_lim@cgh.com.sg

Received: June 29, 2017| Published: August 09, 2017

\section{Introduction}

As the world's population is living longer, there are increasingly more elderly patients requiring surgical intervention. Today, we do more surgeries for the elderly than we used to. The elderly patients receiving surgical treatment are more complex because of their diminished physiological reserve, background co morbidities, cognitive impairment and they are at higher risk of developing postoperative complications and the mortality rate is higher compared to the younger patients. ${ }^{1,2}$ The elderly are predisposed to poor nutrition for multiple reasons. ${ }^{3}$ Among the elderly surgical patients, prevalence of malnutrition was seen in over 50\% and was more prevalent among the elderly admitted for urgent emergency operations than elective operations (69.6\% Vs $34.1 \%)$. The presence of cognitive impairment was a major risk for malnutrition, and depression was not a major risk. The elderly patients who were malnourished tend to be more dependent in their instrumental activities of daily living. ${ }^{4}$ Poor nutrition among the elderly surgical patients is associated with increased postoperative complications, prolonged length of stay and increased healthcare cost. The malnourished elderly with low preoperative body weight, documented weight loss and low serum albumin had a threefold higher mortality compared to the well-nourished group and the high mortality remained persistently high after 3years. ${ }^{5}$ There is a push to encourage early feeding among the elderly surgical patients to improve outcome by limiting postoperative catabolism, maintain immune response and improve wound healing. ${ }^{6}$ This article aims to review the causes of poor nutrition among the elderly surgical patients, the metabolic changes which occur peri operatively and strategies to manage nutrition during the perioperative period for a better overall outcome.

\section{Malnutrition and the elderly}

Malnutrition can either mean over or under nutrition. For the purpose of this paper, malnutrition refers to under nutrition, where the caloric intake is less than daily requirement, with deficiencies of macronutrients (protein) and micronutrients (vitamins and trace elements) resulting in muscle loss and frailty. Among the elderly, prevalence of malnutrition varies between $15 \%$ of community dwelling elderly to $85 \%$ among the elderly in nursing home. The elderly are more likely to have poor dentition, reduced sense of taste and smell which predispose to anorexia and less enjoyment of food. There are social factors which predispose to malnutrition like isolation, food rationing because of difficulties getting supply and financial difficulties. Medical reasons like chronic organ dysfunction, multiple medications, abnormalities of the gastrointestinal tract (GI Tract) like constipation, ulcers, etc may cause anorexia and poor intake. Functional dysphasia is a common finding among the elderly, particularly elderly with neurodegenerative disorders. The elderly with dysphasia may be put on modified diets which are unpalatable and assistance with feeding is often required. The elderly with dementia may have behavioral and mood symptoms which interfere with food intake, particularly if they reside in an institution where meal times are often scheduled and rushed. Assisted feeding takes patience and time among the elderly with dysphasia and dementia, and in an environment where meal times are rushed and staffs are not well trained, malnutrition is common. ${ }^{7,3}$ The elderly surgical patients have different co morbidities and disease profiles for the type of surgical procedures they need. For example, the vascular patients are more likely to have multiple cardiovascular risks and renal disease and are often on restricted diets for their co morbidities. The elderly patients with GI pathology may have dyspepsia, changes in bowel habit and anorexia which predispose to malnutrition. The surgical oncology patients are more likely to be anemic with anorexia, weight loss and cachexia. Because of the high pre-existing risk for malnutrition among the elderly surgical patients, preoperative screening for nutritional status with early intervention is important to improve outcome.

\section{Malnutrition among the elderly oncology patients}

Malignancy is often associated with poor appetite (anorexia) and early satiety. Anorexia is present in up to $50 \%$ of cancer patients and result in poor food intake. Cachexia refers to weight loss due to losses of skeletal muscles and adipose tissues, with abnormalities of electrolyte and water balance. Cachexia is often accompanied by anorexia. The tissue loss due to starvation involves mainly losses of adipose tissues with preservation of skeletal muscle mass, whereas in cancer Cachexia, loss of body mass is predominantly from skeletal muscles causing muscle weakness, immobility and eventual death from respiratory muscle weakness. 
Weight loss in cancer patients is rarely due to poor intake of food alone, hence it is not necessarily associated with a low BMI. This disease related weight loss it is due to changes of body composition with loss of lean muscle mass. In fact, the loss of muscle mass and adipose tissues occurs before reduction in food intake. The caloric intake subsequently decreases to match the lower body weight. The amount of food intake corresponds poorly to the degree of weight loss in cancer patients. Even with adequate dietary counseling and nutritional supplements, adequate food intake is unable to stop the wasting process. Weight gain, if achieved, is temporary and mainly comprises of fat and water, with no increase in lean muscle mass. ${ }^{8,9}$ Anorexia in patients with GI malignancy may be caused by intractable vomiting from intestinal obstruction or dysphasia. Early satiety may be caused by delayed gastric tumour with delayed emptying or tumour encroaching on the GI tract with delayed passage of intestinal content. The hypothalamic food intake centre has receptors for tumour necrosis factor (TNF) and interleukin-1 (IL-1), with close linkage to the serotonergic pathways. Stimulation of the serotonin pathways causes anorexia in rats. Tumours producing TNF and IL-1 act directly to produce anorexi. ${ }^{10}$

\section{Metabolic changes of surgery}

Surgery is a treatment where controlled and deliberate injuries to body parts occur with a cascade of physiological and metabolic changes resulting in catabolism. See Figure 1. Recovery from the surgical procedure requires reversal of metabolism from a catabolic state to anabolic state. Nutritional support is essential in limiting the catabolic state and supporting anabolism.

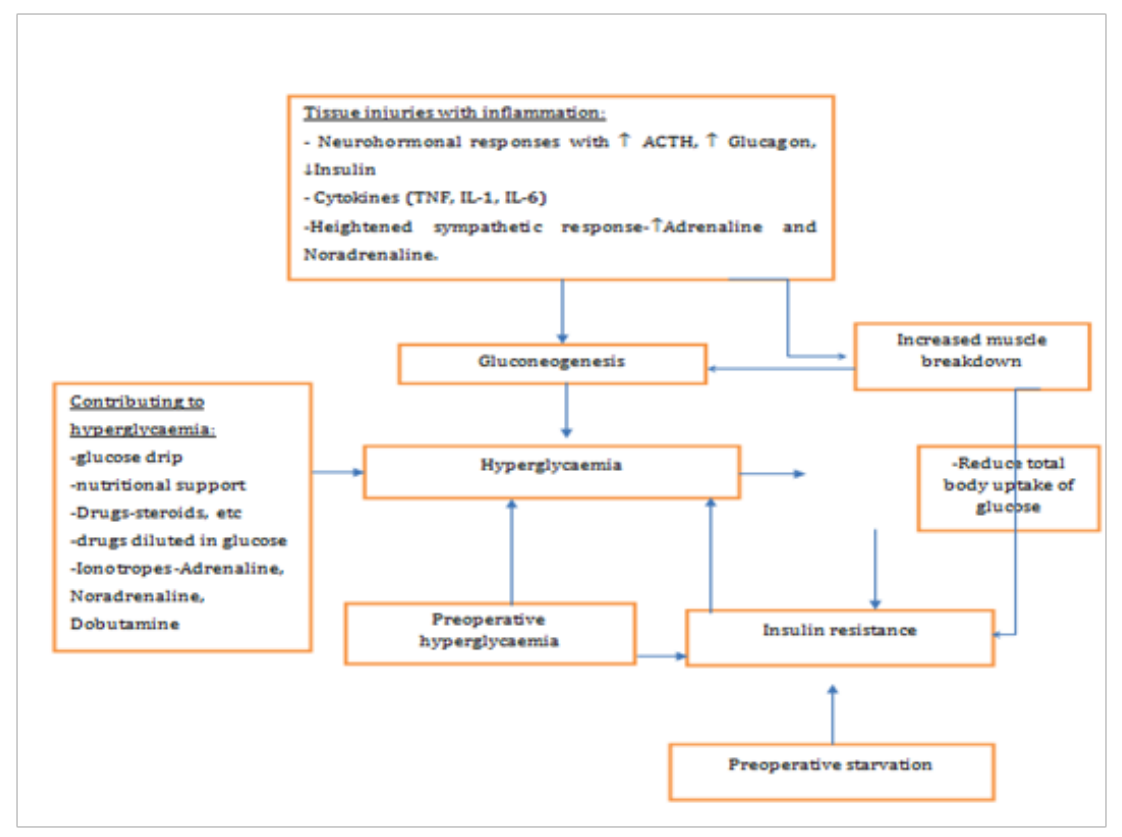

Figure I Diabetes of injury.

\section{Metabolic effects of fasting before surgery}

The patients undergoing gastrointestinal surgery typically undergo prolonged periods of fasting. We are naturally fasted during sleep, with the rest of the day in postprandial state. The fuel we have stored are carbohydrate $(0.4 \mathrm{~kg}$ which lasts for less than a day), protein (10$12 \mathrm{~kg}$ of which only $20-30 \%$ are available as fuel) and fat $(10-15 \mathrm{~kg}$ which can last for 21-55days). Glycogen store in the liver is the main source of glucose, which depletes in a day. After which, protein breakdown from muscles takes over to produce glucose for organs which depend on glucose as a substrate like brain, erythrocytes and renal medulla. Metabolic adaptation occurs within 2-3weeks to avoid excessive muscle breakdown in order to preserve lean muscle mass and function, with breakdown of fat resulting in increasing production of free fatty acids and ketones as fuel. Metabolic rate slows down and insulin secretion reduces. The metabolic adaptation of starvation allows us to survive up to 2 months without food. Once food intake occurs, insulin secretion increases. Insulin is an anabolic agent which causes increased glycogen synthesis, increase peripheral uptake of glucose, mainly in the muscles, reduced glucose production and gluconeogenesis from the liver is inhibited. Brief periods of fasting or hypocaloric intake for 1-3days cause a marked reduction in insulin sensitivity. Insulin resistance is essential to ensure gluconeogenesis, reduce peripheral uptake of glucose and lipolysis to maintain energy production. The low insulin level, with increase in glucagon production leads to reduced Insulin like Growth Factor-1 (IGF-1) activity. IGF-1 is an anabolic hormone which normally increases with protein and carbohydrate intake stimulating amino acids and glucose uptake, as well as protein synthesis by the muscle cells. IGF-1 also causes a drop in serum glucose level. ${ }^{11,12}$ Therefore, the net effects of prolonged starvation result in insulin resistance with reduced glucose uptake by muscles and fat, reduced protein synthesis by muscle cells with muscle loss and breakdown, and breakdown of fat producing free fatty acids and ketones in order to maintain energy production.

It has been a worldwide routine to keep patient starved overnight 
for surgery to minimize aspiration risk during general anesthesia. It has been shown recently that intake of fluids up to 2hours before surgery is safe with no increase in aspiration risk or gastric content. ${ }^{13}$ To improve the metabolic state during the surgery, a carbohydrate drink ( $400 \mathrm{ml} 50 \mathrm{~g}$ glucose) taken at $2-3 \mathrm{hr}$ before surgery, was shown to have enough calorie to stimulate insulin to levels seen after a meal. As a result, insulin resistance reduces by about $50 \%$ postoperatively when preoperative fasting is avoided. The increased insulin release improves peripheral uptake of glucose and less production of glucose. $^{14}$

\section{Perioperative catabolism}

\section{Glucose metabolism and insulin resistance}

Within hours after initiating surgery, insulin resistance develops due to tissue injury. The degree of insulin resistance is proportional to the magnitude of surgery. The metabolic rate increases and catabolism with breakdown of glycogen, fat and protein occurs. The tissue injuries which occur during surgery causes a series of endocrineinflammatory changes in the perioperative period resulting in increased gluconeogenesis from increased release of catecholamines, glucagon, ACTH and cytokines. Serum glucose level is often high as a result, and the peripheral uptake of glucose is impaired, with low basal levels of insulin causing insulin resistance. The changes in glucose metabolism are similar to untreated diabetes. Hyperglycaemia is further contributed by infusion of glucose drip, and overfeeding with high carbohydrate loads. ${ }^{15}$ Unlike starvation, there is no metabolic adaptation to reduce loss of lean body mass postoperatively.

Insulin resistance postoperatively is a predictor of adverse events, with longer length of stay. For every $20 \%$ reduction in insulin sensitivity, the risks of mortality, heart failure, stroke, dialysis need and infection among the cardiac surgical patients more than doubled, irrespective of their diabetes status. ${ }^{16,17}$ Surgical techniques with less tissue injury like laparoscopic procedures have less impact on glucose metabolism compared to laparotomy..$^{18}$ In elective surgeries, all the metabolic changes described can be reversed by insulin. In the postoperative period, several fold higher doses of insulin is required to normalize glucose level and reducing breakdown of lean body mass which suggest presence of insulin resistance in the postoperative period. ${ }^{19}$

\section{Protein catabolism}

Loss of muscle mass occurs early and rapidly during stress and is more severe among patients with multi-organ failure. For a healthy adult, an elective abdominal surgery causes a loss of $1.2-2.4 \mathrm{~kg}$ of skeletal muscle mass. Patients with burns and sepsis lose up to $800 \mathrm{~g}$ per day of muscle mass. For the diabetic patients undergoing colorectal cancer surgery, their losses can be $50 \%$ higher than in non-diabetics. ${ }^{20,21}$ The elderly show similar magnitude of protein catabolism and muscle breakdown. The elderly are even more vulnerable to losses of muscle mass and the resultant physical disability and functional decline, as they often have pre-existing sarcopenia due to the ageing process, malnutrition and low physical activities. ${ }^{3,22}$

\section{Postoperative fasting}

The key objectives to early recovery are to have early return to preoperative level of functioning and well-being. Insulin resistance which was initiated during the preoperative period persists in the postoperative period causing ineffective glucose transport into the muscle cells, continual loss of protein from muscles and less glycogen synthesis. These result in loss of muscle bulk and strength with functional decline. Bed rest and persistence of low oral food intake in the postoperative period further contribute to insulin resistance and loss of muscle strength. Metabolically, the postoperative period aims at returning the patient to the anabolic state from the catabolism of surgery with early feeding and mobilization. With early feeding in the postoperative period, insulin release is increased by about 6-8 times which stimulates uptake of glucose in the periphery and shuts off gluconeogenesis. Artificial feeding via enteral or parenteral routes may be necessary in some postoperative cases, if the oral intake is insufficient to meet the caloric requirements prescribed. ${ }^{15}$

\section{Strategies to enhance overall recovery after surgery}

There is increasing awareness to reduce the stress of surgery, with support to enhance anabolism and minimize catabolism in order to enhance postoperative recovery. The programme for Fast Tract Surgery developed into Enhanced Recovery after Surgery (ERAS), using a multimodal and multidisciplinary care approach. ERAS was developed for elective colon surgeries but has now extended to include patients for other major surgeries such as rectal, gynecological, liver, upper gastrointestinal and urological surgeries. The aims of ERAS are to improve overall surgical outcome focusing on nutritional support, pain management and early mobilization to support early return of function. ERAS has been shown to be safe and beneficial for the elderly. The recommended measures and rationale are listed in Table 1. To be effective, patients need to receive a combination of all these measures, rather than how each component is done. ${ }^{23,24}$

\section{Strategies for management of nutrition}

Selected components of ERAS which are relevant to the nutritional support for the elderly will be mentioned below in greater details. These measures are important not only to improve the elderly surgical patients' nutritional status, improve their metabolic profile but also to enhance their overall recovery from surgery.

\section{Preoperative fasting and carbohydrate loading}

There has been a shift in practice in the last decade to reduce the preoperative fasting period to 2-3hours prior to anesthesia for elective surgery. The patients are encouraged to eat normally up until the evening before surgery, in addition to a carbohydrate loaded drink before anesthesia. This change has been shown to be safe with no increase in aspiration risk, except for emergency surgeries or patients with delayed gastric emptying. Keeping the patient in a fed state increases the body's insulin secretion and reduces the hyperglycaemia and insulin resistance state postoperatively.

There is now a trend towards giving an iso-osmolar carbohydrate rich drink on the evening before surgery and one at up to 2-3hours before anesthesia. This strategy has been proven to be safe as the fluid is rapidly cleared from the stomach and it reduces patients' discomfort for thirst, hunger, anxiety and reduces risk of dehydration. More importantly, this strategy brings the patient to surgery in a fed state, rather than a state of starvation which improves insulin sensitivity and preserve skeletal muscle mass in the postoperative period. Thus, the patient is in an anabolic state with more benefit to maintain uptake of glucose in the periphery and maintain lean body mass from postoperative nutrition. ${ }^{25}$ The current recommendation for fasting is therefore 6-hour solid fast, and intake of clear fluids up to 2hours before initiation of anesthesia. For the patients excluded from the oral 
carbohydrate drink preoperatively, an intravenous infusion of glucose with or without insulin produces the same effect, with $5 \mathrm{mg} / \mathrm{kg} / \mathrm{min}$ of glucose infusion. ${ }^{15}$

\section{Preoperative bowel preparation}

For patients undergoing bowel resection, it was traditional to clear the bowels of faecal material with an aim to reduce the risk of postoperative infection should an anastomotic leak occur. Technically, an empty colon also makes laparoscopic surgery easier to manipulate and an empty colon makes it easier for the surgeon to palpate for the colonic tumour. The collapsed colon is also less likely to develop ischaemia from pressure due to excessive stretch by a faecally loaded colon. However, bowel cleansing has not been shown to be useful. On the contrary, it increases postoperative infection, showed no difference in anastomotic leak compared to the patients not prescribed colonic cleansing. For the patients who did not have preoperative cleansing, they have a shorter LOS and earlier return of bowel function. ${ }^{26}$ Bowel preparation is stressful for the patients particularly the elderly, who may develop dehydration with fluid and electrolyte abnormalities..$^{27}$ Nonetheless, for the patients who are required to undergo preoperative bowel cleansing, they are encouraged to take oral nutritional supplements (ONS) during or after the cleansing to reduce the overall preoperative fasting period and the absorption of nutrients is not compromised by bowel cleansing. ${ }^{15}$

Table I components of ERAS ${ }^{23}$

\begin{tabular}{|c|c|}
\hline Main elements & Recommendations \\
\hline $\begin{array}{l}\text { Preadmission counseling and } \\
\text { information }\end{array}$ & $\begin{array}{l}\text { Written and oral instructions with clear roles on specific tasks during the perioperative period to } \\
\text { improve adherence to care pathway. }\end{array}$ \\
\hline Preoperative bowel preparation & $\begin{array}{l}\text { Not indicated unless colonoscopy is required during op. Stressful for patients, especially the elderly and } \\
\text { have not been shown to reduce infections. }\end{array}$ \\
\hline Preoperative fasting & $\begin{array}{l}\text { No evidence that overnight fast reduce aspiration risk. Current recommendations- } 6 \text {-h fast for solid food } \\
\text { and clear fluids up to } 2 \mathrm{~h} \text { before anesthesia. }\end{array}$ \\
\hline $\begin{array}{l}\text { Preanaesthetic anxiolytic and } \\
\text { analgesia }\end{array}$ & Not proven to be useful, cause postoperative sedation except for patients with long term sedative use. \\
\hline $\begin{array}{l}\text { Prophylaxis for venous } \\
\text { thrombosis }\end{array}$ & $\begin{array}{l}\text { Low molecular weight heparin preferred. Start at } 2-12 \mathrm{~h} \text { before surgery until patient is fully mobile, with } \\
\text { compression stockings. }\end{array}$ \\
\hline Antibiotic prophylaxis & $\begin{array}{l}\text { Single dose prophylaxis against aerobic and anaerobic organisms, before skin incision and intraop if op } \\
\text { is }>3 \mathrm{~h} \text {. }\end{array}$ \\
\hline Anesthetic agents & Short acting anesthetic drugs and analgesia to allow early recovery. \\
\hline Surgical incisions & $\begin{array}{l}\text { No consensus on transverse/curved Vs longitudinal incisions for colorectal op. Recommendation- } \\
\text { minimizes length of incision. }\end{array}$ \\
\hline $\begin{array}{l}\text { Nasogastric tube for } \\
\text { decompression }\end{array}$ & Not routinely recommended, increased risk of fever, atelectasis and pneumonia. \\
\hline $\begin{array}{l}\text { Maintain Intraoperative } \\
\text { normothermia }\end{array}$ & $\begin{array}{l}\text { Improves endocrine-metabolic profile, reduces bleeding, transfusion requirements, wound infections } \\
\text { and cardiac complications. }\end{array}$ \\
\hline Perioperative IV fluids & Maintain fluid balance and avoid overload. Commence oral fluids $2 \mathrm{~h}$ post surgery. \\
\hline Drainage of peritoneal cavity & Not routinely recommended as it impedes on mobility, with no proven benefits. \\
\hline Urinary drainage & $\begin{array}{l}\text { Recommended only for the duration of thoracic epidural. Earliest removal of catheter to facilitate } \\
\text { mobility. }\end{array}$ \\
\hline $\begin{array}{l}\text { Postoperative nausea and } \\
\text { vomiting }\end{array}$ & Avoid drugs which are emetogenic, use antiemetic selectively, aim to achieve early feeding. \\
\hline Postoperative analgesia & $\begin{array}{l}\text { Epidural, NSAIDS, paracetamol in combination to achieve good analgesic effects to aid early } \\
\text { mobilization. }\end{array}$ \\
\hline Postoperative feeding & $\begin{array}{l}\text { Patients should be encouraged to eat orally } 4 \text { hour after surgery. ONS should be taken from the day of } \\
\text { surgery until oral intake achieves normal level. For the malnourished patients, longer preop ONS to be } \\
\text { followed by longer postpone ONS. }\end{array}$ \\
\hline Early mobilisation & Being out of bed for $2 \mathrm{~h}$ on the day of surgery and $6 \mathrm{~h}$ there after \\
\hline
\end{tabular}




\section{Perioperative fluid management}

Intravenous fluid therapy is given a special mention as it is considered as a medical intervention to support fluid and electrolyte balance. Abnormalities of fluid and electrolytes affect the gastrointestinal function and have an indirect effect on nutrition. There is increasing evidence to show that excessive intravenous infusion of saline is associated with poor outcome such as delayed return of gastrointestinal function, cardiopulmonary congestion, and delayed wound healing and longer length of hospital stay. ${ }^{28}$ The traditional teaching of aggressive fluid replacement intraoperatively to support evaporative loss from the abdominal cavity during surgery, fluid loss into the third space and fluid accumulated in traumatized tissues were not evidence based and overestimated. The current recommendation is to replace only the estimated fluid loss or use as maintenance therapy and fluid overload should be avoided. ${ }^{29}$ The elderly surgical patients are particularly susceptible to develop fluid overload and cardiopulmonary congestion with intravenous fluid therapy during the perioperative period because of their poor cardiac reserve. During the acute phase of surgery, there is increased release of $\mathrm{ADH}$, coupled with heightened sympathetic response which leads to increased rennin and aldosterone response where water and salt retention is exacerbated. ${ }^{30}$

Excess salt and water retention delays return of gastrointestinal function by causing intestinal edema and raised intra-abdominal pressure which compromises mesenteric perfusion. The salt and water overload, together with mesenteric ischaemia, increases risk of anastomotic leak, delay in gastric emptying and postoperative ileus. ${ }^{31}$ Postoperative ileus has negative effects on the patients with persistent pain and discomfort, increase pulmonary complications and enhances catabolism because of poor nutrition, and as a result, increases length of stay. ${ }^{32}$ Strategies to reduce postoperative ileus include epidural anesthesia, avoidance of opioids, fluid overload and oral magnesium oxide ( $1 \mathrm{~g}$ BD on evening of surgery until discharge).$^{23}$

\section{Postoperative oral nutritional intake}

The traditional management of elective GI surgeries was placement of nasogastric tube (NGT) for decompression and keeping the patients nil by mouth (NBM) until they pass flatus. The rationale for this practice was to allow time for anastomoses to heal and to reduce postoperative nausea and vomiting due to gastric distension. There is now increasing trend to avoid NGT insertion and allowing oral feeding early postoperatively. There are data available to avoid keeping the patients NBM after major Upper GI, cholecystectomy, colorectal resection, gynecological, urological and vascular surgeries. ${ }^{33}$ Early oral food intake or oral nutritional supplement (ONS) on postpone day 1 or 2 does not cause increased anastomotic leaks and has been proven to be beneficial in shortening length of stay, early postoperative recovery, resumption of bowel movement, improved outcomes like mortality and morbidity. Early postoperative feeding is one of the measures in ERAS and has been shown to be safe after elective colorectal surgery with shortened hospital stay. ${ }^{23} \mathrm{~A}$ free diet initiated after surgery led to earlier tolerance of normal diet at postpone day 2, without having to insert NGT, with no difference in postoperative ileus. For gastrectomy, omission of naso-jejunostomy tubes for oral feeding led to shorter length of stay. ${ }^{34,35}$

\section{Perioperative nutritional support}

Nutritional support is nutrient support either by diet (fortified food and drinks or oral nutrition supplement) which can either be given enterally or parenterally. Nutritional support is usually individualized and includes dietary counseling and advice.

The key aspects of perioperative nutrition care include:

i. Identify nutrition risk and integrate intervention into general management early.

ii. Avoid prolonged fasting.

iii. Re-establish oral feeding as early as possible, after surgery.

iv. Metabolic control of blood glucose, fluid and electrolytes.

v. Identify and remove factors which exacerbate catabolism or impair return of GI function.

vi. Early mobilization to facilitate protein synthesis and muscle function.

The main aims of nutritional support in surgical patients are to reduce catabolism and malnutrition. The indicators of nutritional support include improved quality of life and return of function postoperatively. Nutritional support is not only indicated for patients who are malnourished. It should be considered early if the surgical patient is anticipated to have inadequate or no oral intake for more than 5days perioperatively, or the patients who are unable to maintain above $50 \%$ of their recommended amount of oral intake for more than 7 days. It is recommended to start nutrition support as soon as risks are identified, rather than wait until malnutrition has occurred. Parenteral nutrition is required if oral and enteral route of intake cannot meet $>50 \%$ of requirement for more than 7 days. Enteral nutrition support is the preferred method of supplementation. Parenteral nutrition is considered only if the GI tract is not functioning and should be started early if nutritional support is indicated..$^{33}$ For the elderly patients, it is recommended to do a comprehensive geriatric assessment on admission which will indicate the presence of nutritional risks, particularly weight loss $>5 \%$ in 3 months, reduced food intake, disease severity and $\mathrm{BMI}<20.5 \mathrm{~kg} / \mathrm{m}^{2}$. ${ }^{36}$

\section{Preoperative nutritional support}

For the elderly general surgical patients, two parameters, weight loss of $>10 \%$ in previous 6 months and low serum albumin are reliable predictors of nutritional status and are associated with postoperative outcome. ${ }^{37}$ The rationale for preoperative nutritional support is treatment and prevention of malnutrition as surgery often involves prolonged periods of fasting and catabolism. ${ }^{33}$

For the mildly malnourished patients or patients with nutritional risk, short term preoperative nutritional support of 7-10days is recommended. For the severely malnourished patients, longer period of nutritional support is required, in combination with resistance training. Surgery may be postponed to improve the risks unless it is an urgent procedure. For the patients with severe malnutrition, surgical procedures should be limited to minimize tissue injuries in order to reduce stress of surgery and minimize catabolism. ${ }^{33}$ For the patients with significant deficit preoperatively, several weeks of supplementation before surgery is ideal to replace the deficit, but is seldom tolerated for 
various reasons. The elderly with sarcopenia are considered high risk. For the patients with severe nutrition risk, malnutrition or are unable to consume the caloric requirements enterally, parenteral nutrition for 7-14days preoperatively is recommended..$^{33}$

\section{Postoperative nutritional support}

The surgeon has to balance the extent of surgery against wound healing for the severely malnourished patients with loss of lean body mass (LBM). LBM is $50-60 \%$ muscle mass by weight. The severely malnourished patients, may exhibit poor immune response to tissue injuries with poor wound healing. The greater the lossof LBM, the longer it takes for the wound to heal. With a loss of $20 \% \mathrm{LBM}$, there is equal competition for the dietary protein ingested for wound to heal and for the body to restore the LBM. Losses of LBM of $>30 \%$ cause spontaneous wounds like pressure sores to develop and there is a threat to survival. Therefore, among the patients with losses of LBM of $>30 \%$, the dietary protein will be used to restore LBM, as a priority and the wound essentially stop healing until the LBM is partially restored. The nutritional support for patients with changes in body composition, particularly loss of LBM, prior to surgery requires overall increase in caloric content as well as protein content. The average adults consume $0.8-1.0 \mathrm{~g} / \mathrm{kg} / \mathrm{day}$ of protein and surgical patients with wounds may need about $1.5 \mathrm{~g} / \mathrm{kg} / \mathrm{day} .{ }^{3,38}$ For the severely malnourished elderly, there is clear advantage for the prescription of ONS in the immediate postoperative period and the benefit extend up to 8 th postoperative week, with positive outcome observed for recovery of nutritional status, positive protein balance and quality of life. With the current recommendations by ERAS, preoperative ONS prescription, with continuation within 4days postoperatively result in better nitrogen balance without the risk of hyperglycaemia. This shows improvement in the metabolic control and improvement in insulin resistance in the perioperative period. ${ }^{39,40}$ For the patients who are unable to take orally such as major head and neck or neurosurgical patients or patients who are unable to take $50 \%$ of their caloric needs for more than 7 days, early tube feeding is recommended. ${ }^{33}$

\section{Conclusion}

The elderly surgical patients are complex and challenging to care for, compared to the younger patients because of their multiple co morbidities and poor reserve. Poor nutrition is common among the elderly and is often not actively screened for by the healthcare workers. Malnutrition and weight loss among the surgical patients are predictors of poor outcome, mortality and morbidity. The extent of surgery is important to minimize further catabolism of their lean body mass and prevent further functional decline postoperatively. Nutritional intervention, together with ERAS in the perioperative period has been shown to be beneficial and has made significant advances in the recent years.

\section{Acknowledgements}

None.

\section{Conflict of interest}

The author declares no conflict of interest

\section{References}

1. Durron JJ, Duron E, Dugue T, et al. Risk factors for mortality in major digestive surgery in the elderly: a multicenter prospective study. Ann Surg. 2011;254(2):375-382
2. Massaeweh NN, Legner VJ, Symons RG, et al. Impact of advancing age on abdominal surgical outcomes. Arch surg. 2009;144(12):1108-1114.

3. SC Lim. Nutrition and the role of tube feeding in the elderly. J JGeronto. 2016;2(1):017.

4. Damuleviciene G. Nutritional status of elderly surgical patients. Medicine (Kaunas). 2008;44(8):609-618.

5. Gregg J. Effect of preoperative nutritional deficiency on mortality after radical cystectomy for bladder cancer. J Urol. 2011;185(1):90-96.

6. Braga M, Gianotti L, Nespoli L, et al. Nutritional approach in malnourished surgical patients. Arch Surg. 2002;137(2):174-180.

7. Morley JE. Death by starvation, A modern American problem? $J \mathrm{Am}$ Geriatr Soc. 1989;37(2):184-185.

8. Costa G, Lane WW, Vincint RG, et al. (1980) Weight loss and cachexia in lung cancer. Nutr Cancer 2: 985.

9. Evans WK, Makuch R, Clamon GH, et al. Limited impact of total parenteral nutrition on nutritional status during treatment for small cell lung cancer. Cancer Res. 1985;45(7):3347

10. Michael J Tisdale. Cancer anorexia and cachexia. Nutrition. 2001;17(5):438-442.

11. Jonas Nygren. The metabolic effects of fasting and surgery. Best Prac and research ClinAnaesth. 2006;20(3):429-438.

12. Clemmons DR. Nutritional regulation of IGF-1 and IGF binding proteins. Annu Rev Nutr. 1991;11:393-412.

13. Brady M. Preoperative fasting for adults to prevent perioperative complications. Cochrane Database of Syst Rev. 2003;4:CD00423.

14. Nygren J, Soop M, Thorell A, at al. preoperative oral carbohydrate administration reduces postoperative insulin resistance. Clinical Nutrition. 1998;17(2):65-71.

15. Olle Ljungqvist. Basics in Clinical Nutrition: Perioperative nutrition. E-SPEN. The European e-Journal of Clinical Nutrition and Metabolism. 2010;5(2):e93-96.

16. Thomas Schricker, Ralph Lattermann. Perioperative catabolism. Can J Anaesth. 2015;62(2):182-193.

17. Sato H, Carvalho G, Sato $T$, et al. The association of perioperative glycaemic control, intraoperative insulin sensitivity, and outcomes after cardiac surgery. J Clin Endocrinol Metab. 2010;95(99):4338-4344.

18. Carli F, Galeone M, Gzodzic B, et al. Effect of laparoscopic colon resection on postoperative glucose utilization and proteinsparing: an integrated analysis of glucose and protein metabolism during the fasted and fed States using stable isotopes. Arch Surg. 2005;140(6):59359-59367.

19. Ljungqvist O, Fearon K, Little RA. Clinical nutrition. In: Gibney MJ, et al. editors. The Human Nutrition Textbook Series. Oxford, UK: Blackwell Science; 2005.

20. Kinney JM, Elwyn DH. Protein metabolism and injury. Annu rev Nutr. $1983 ; 3: 433-466$

21. Schricker T, Gougeon R, Eberhart L, et al. Type 2 Diabetes Mellitus and the catabolic response to surgery. Anaesthesiology. 2005;102(2):320-326.

22. Morais JA. Protein turnover and requirements in the healthy and frail elderly. J Nutr Health Aging. 2006;10(4):272-283.

23. KCH Fearon, Ljungqvist O, Von Meyenfeldt M, et al. Enhanced recovery after surgery: A consensus review of clinical care of patients undergoing colonic resection. Clin Nutr. 2005;24(3):466-477.

24. Slieker J, Frauche P, Jurt J, et al. Enhanced Recovery ERAS for elderly: a safe and beneficial pathway in colorectal surgery. Int $J$ Colorectal Dis. 2017;32(2):215-521 
25. Yuill KA, Richardson RA, Davidson HI, et al. The administration of an oral carbohydrate-containing fluid prior to major elective uppergastrointestinal surgery preserves skeletal muscle mass postoperatively-a randomised clinical trial. Clin Nutr. 2005;24(1):32-37.

26. Anjali S Kumar, Kelleher DC, Sigle GW, et al. Bowel preparation before elective surgery. Clin Colon Rectal Surg. 2013;26(3):146-152.

27. Holte K, Nielsen KG, Madsen JL, et al. Physiologic effects of bowe preparation. Dis Colon rectum. 2004;47(8):1397-1402.

28. Brandstrup B, Tønnesen H, Beier-Holgersen R, et al. Effects of intravenous fluid restriction on postoperative complications: comparison of two perioperative fluid regimens: a randomised assessor blinded multicentre trial. Ann Surg. 2003;238(5):641-648.

29. Brandstrup B. Fluid therapy for the surgical patient. Best Practice \& Research Clinical Anasesthesiology. 2006;20(2):265-283.

30. Desborough JP. The stress response to trauma and surgery. Br J Anaesth. 2000;85(1):109-117.

31. Chowdhury AH. Fluids and the gastrointestinal function. Curr opin Clin Nutr and Metab Care. 2011;14(5):469-476.

32. Barletta JF. reducing the burden of postoperative ileus: evaluating and implementing an evidence-based strategy. World J Surg. 2014;38(8):19661977.
33. Arved Weimann, Braga M, Carli F, et al. ESPEN guideline: Clinical nutrition in surgery. Clin Nutr. 2017;36(3):623-650.

34. Han-Geurts IJ, Hop WC, Kok NF, et al. Randomised clinical trial of the impact of early enteral feeding on postoperative ileus and recovery. $\mathrm{Br} J$ Surg. 2007;94(4):555-561

35. Carrere N. Is nasogastric or nasojenunal decompression necessary after gastrectomy? A prospective randomised trial. World J surg. 2007;31(1):122-127.

36. Cheema FN. Novel approach to perioperative assessment and intervention may improve long term outcomes after colorectal cancer resection in older adults. Ann Surg. 2011;253(5):867-874.

37. Mireille FM. Preoperative nutrition status and postoperative outcome in elderly surgical patients: A systematic review. JPEN J of parenter Enteral Nutr. 2013;37(1):37-43.

38. Robert H Demling. Nutrition, anabolism and the wound healing process: an overview. Eplasty. 2009;9:e9.

39. Soop M, Carlson GL, Hopkinson J, et al. Randomised control trial of the effects of immediate enteral nutrition on metabolic responses to major colorectal surgery in an enhanced recovery protocol. $\mathrm{Br} J$ Surg. 2004;91(9):1138-1145.

40. Fearon KC, Luff $\mathrm{R}$. The nutritional management of surgical patients: enhanced recovery after surgery. Proc Nutr Soc. 2003;62(4):807-811. 